\title{
Synthesis, characterization and antimicrobial activity of some 4-aryl-2,6-di(coumarin-3-yl)pyridines
}

\author{
Anil K. Patel, Niraj H. Patel, Mehul A. Patel and Dinker I. Brahmbhatt* \\ Department of Chemistry, Sardar Patel University, Vallabh Vidyanagar-388 120, Gujarat, India \\ E-mail: drdibrahmbhatt@gmail.com
}

\begin{abstract}
Some 4-aryl-2,6-di(coumarin-3-yl)pyridines 3a-r are synthesized by the reaction of 3coumarinoyl methyl pyridinium salts 1a-c with appropriate 1-[2H-1-benzopyran-2-on-3-yl]-3aryl-prop-2-en-1-ones 2a-f in the presence of ammonium acetate and acetic acid under the Kröhnke reaction conditions. All the synthesized compounds are screened for antimicrobial activity.
\end{abstract}

Keywords: Coumarins, dicoumarinyl pyridines, pyridylcoumarins, Kröhnke reaction, antimicrobial activity

\section{Introduction}

Coumarins constitute an important class of benzopyrones, exhibiting a broad range of biological activities such as anticoagulants, ${ }^{1}$ antimicrobial, ${ }^{2}$ antibacterial, ${ }^{3}$ anticancer, ${ }^{4}$ and anti-HIV activity. ${ }^{5}$ The interesting biological activities of the coumarins make them attractive targets in organic synthesis. Coumarins having pyridine substitution at C-3 are reported to have interesting biological activity. Many 3-(2-pyridyl)- and 3-(3-pyridyl)coumarins are known for their useful bioactivities viz. antifungal, ${ }^{6,9}$ bactericidal, $^{7}$ fish toxicity ${ }^{7}$ and moth proofing activity. ${ }^{8}$ Some of them are also known for their CNS depressant activity. ${ }^{9}$ Considering their biological importance, a variety of 3-pyridyl substituted coumarins were earlier synthesized from our laboratory. ${ }^{10}$ In continuation of this interest, we now report the synthesis of 4-aryl-2,6-di(coumarin-3yl)pyridines 3. The pyridine nucleus in these compounds is flanked by two coumarin moieties at C-2 and C-6 and the structure seems as if two coumarins have 3-(2-pyridyl) substitution. One can expect improved biological potency for such compounds.

During our literature search on the synthesis of 2,6-dicoumarinyl pyridine derivatives, we came across to two reports in which this type of compound had been synthesized. El-Taweel et al. ${ }^{11}$ synthesized 2,6-di(coumarin-3-yl)pyridine (A) by reacting enaminones of 3-acetylcoumarin with 3-acetylcoumarin in the presence of ammonium acetate in refluxing acetic acid. However, 
the main objective of these authors was not to synthesize dicoumarinyl pyridines, but to study the reaction of enaminones of 3-acetylcoumarin with variety of reagents and to prepare various heterocyclic substituted coumarins. Only one dicoumarinyl pyridine (A) was prepared in this work. Verma et al. ${ }^{12}$ synthesized various 4-aryl-2,6-dicoumarinyl pyridines (B) by the condensation of 1-[2H-1-benzopyran-2-on-3-yl]-3-aryl-prop-2-en-1-ones with urea or amide derivatives using bismuth(III) nitrate- $\mathrm{Al}_{2} \mathrm{O}_{3}$ as a catalyst. However though the method is simple and convenient, the main drawback of the method is that only symmetrically substituted 2,6dicoumarinyl compounds can be prepared, i.e. the product will have identical coumarin moieties at $\mathrm{C}-2$ and $\mathrm{C}-6$ of the pyridine.

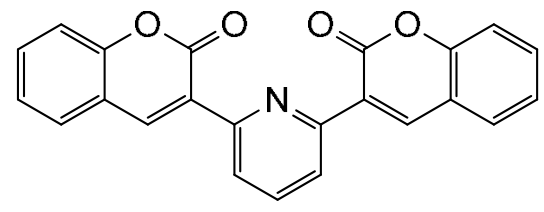

[A]

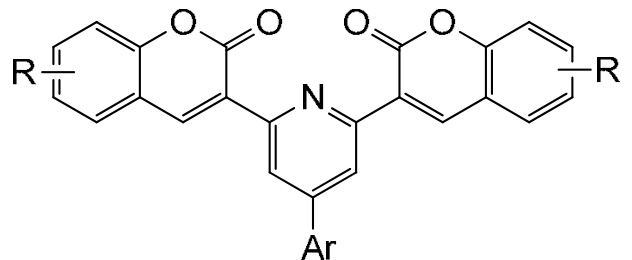

$[\mathrm{B}]$

Considering the above facts, in the present work we have developed a simple, convenient and general method for the synthesis of 4-aryl-2,6-dicoumarin substituted pyridines utilizing easily accessible starting materials. The method involves the condensation of 3-coumarinoyl methyl pyridinium salts with 1-[2H-1-benzopyran-2-on-3-yl]-3-aryl-prop-2-en-1-ones under Kröhnke reaction conditions. By this method, symmetrically as well as asymmetrically 2,6-dicoumarinyl substituted pyridines can be synthesized.

\section{Results and Discussion}

In the present work, various 4-aryl-2,6-di(coumarin-3-yl)pyridines 3a-r have been synthesized by reacting 3-coumarinoyl methyl pyridinium salts 1a-c with appropriate 1-[2H-1-benzopyran-2on-3-yl]-3-aryl-prop-2-en-1-ones 2a-f in the presence of ammonium acetate and acetic acid under Kröhnke reaction conditions ${ }^{13}$ (Scheme 1). 
<smiles>[R10]c1ccc(/C=C/C(=O)c2cc3cccc([R3])c3oc2=O)cc1</smiles>

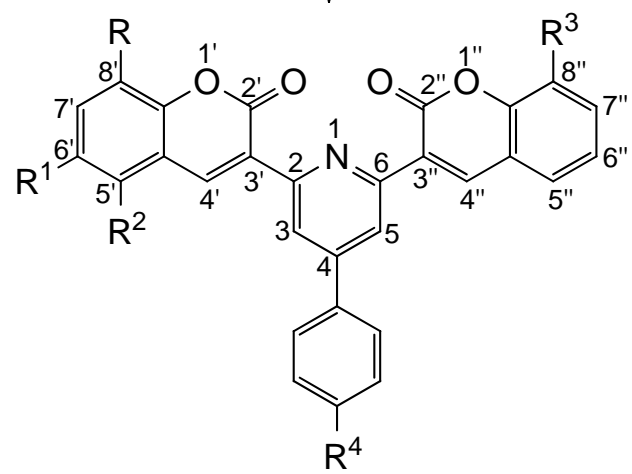

(3a-r)

\begin{tabular}{cccccccccccc}
\hline & $\mathrm{R}$ & $\mathrm{R}^{1}$ & $\mathrm{R}^{2}$ & $\mathrm{R}^{3}$ & $\mathrm{R}^{4}$ & & $\mathrm{R}$ & $\mathrm{R}^{1}$ & $\mathrm{R}^{2}$ & $\mathrm{R}^{3}$ & $\mathrm{R}^{4}$ \\
\hline $\mathbf{3 a}$ & $\mathrm{H}$ & $\mathrm{H}$ & $\mathrm{H}$ & $\mathrm{H}$ & $\mathrm{H}$ & $\mathbf{3 j}$ & $\mathrm{OCH}_{3}$ & $\mathrm{H}$ & $\mathrm{H}$ & $\mathrm{OCH}_{3}$ & $\mathrm{H}$ \\
$\mathbf{3 b}$ & $\mathrm{H}$ & $\mathrm{H}$ & $\mathrm{H}$ & $\mathrm{H}$ & $\mathrm{CH}_{3}$ & $\mathbf{3 k}$ & $\mathrm{OCH}_{3}$ & $\mathrm{H}$ & $\mathrm{H}$ & $\mathrm{OCH}_{3}$ & $\mathrm{CH}_{3}$ \\
$\mathbf{3 c}$ & $\mathrm{H}$ & $\mathrm{H}$ & $\mathrm{H}$ & $\mathrm{H}$ & $\mathrm{OCH}_{3}$ & $\mathbf{3 l}$ & $\mathrm{OCH}_{3}$ & $\mathrm{H}$ & $\mathrm{H}$ & $\mathrm{OCH}_{3}$ & $\mathrm{OCH}_{3}$ \\
$\mathbf{3 d}$ & $\mathrm{H}$ & $\mathrm{H}$ & $\mathrm{H}$ & $\mathrm{OCH}_{3}$ & $\mathrm{H}$ & $\mathbf{3 m}$ & $\mathrm{H}$ & $\mathrm{Benzo}$ & $\mathrm{H}$ & $\mathrm{H}$ \\
3e & $\mathrm{H}$ & $\mathrm{H}$ & $\mathrm{H}$ & $\mathrm{OCH}_{3}$ & $\mathrm{CH}_{3}$ & $\mathbf{3 n}$ & $\mathrm{H}$ & $\mathrm{Benzo}$ & $\mathrm{H}$ & $\mathrm{CH}_{3}$ \\
$\mathbf{3 f}$ & $\mathrm{H}$ & $\mathrm{H}$ & $\mathrm{H}$ & $\mathrm{OCH}_{3}$ & $\mathrm{OCH}_{3}$ & $\mathbf{3 o}$ & $\mathrm{H}$ & $\mathrm{Benzo}$ & $\mathrm{H}$ & $\mathrm{OCH}_{3}$ \\
$\mathbf{3 g}$ & $\mathrm{OCH}_{3}$ & $\mathrm{H}$ & $\mathrm{H}$ & $\mathrm{H}$ & $\mathrm{H}$ & $\mathbf{3 p}$ & $\mathrm{H}$ & $\mathrm{Benzo}$ & $\mathrm{OCH}_{3}$ & $\mathrm{H}$ \\
$\mathbf{3 h}$ & $\mathrm{OCH}_{3}$ & $\mathrm{H}$ & $\mathrm{H}$ & $\mathrm{H}$ & $\mathrm{CH}_{3}$ & $\mathbf{3 q}$ & $\mathrm{H}$ & $\mathrm{Benzo}$ & $\mathrm{OCH}_{3}$ & $\mathrm{CH}_{3}$ \\
3i & $\mathrm{OCH}_{3}$ & $\mathrm{H}$ & $\mathrm{H}$ & $\mathrm{H}$ & $\mathrm{OCH}_{3}$ & $\mathbf{3 r}$ & $\mathrm{H}$ & $\mathrm{Benzo}$ & $\mathrm{OCH}_{3}$ & $\mathrm{OCH}_{3}$ \\
\hline
\end{tabular}

Scheme 1. Synthetic scheme for 4-aryl-2,6-di(coumarin-3-yl)pyridines 3a-r.

The reactions proceeded smoothly and gave the expected products 3a-r in moderate yields (55-66\%). The structures of the compounds 3a-r were established on the basis of $\mathrm{IR},{ }^{1} \mathrm{H},{ }^{13} \mathrm{C}$, DEPT NMR and elemental analysis.

In the IR spectra, compounds 3a-r showed a very strong band between $1713-1730 \mathrm{~cm}^{-1}$ for $\delta$ lactone carbonyl $(\mathrm{C}=\mathrm{O})$ stretching vibrations. The strong bands for aromatic $\mathrm{C}=\mathrm{C}$ and $\mathrm{C}=\mathrm{N}$ stretching vibrations, were observed between $1595-1610$ and $1495-1560 \mathrm{~cm}^{-1}$ respectively. The aromatic C-H stretching vibrations were observed between $3050-3060 \mathrm{~cm}^{-1}$ in the form of a medium band. 
In the ${ }^{1} \mathrm{H}$ NMR spectra of compounds 3a-r, the aromatic protons appeared in the form of multiplets between $\delta 7.02-8.59$. The pyridine $3-\mathrm{H}$ and $5-\mathrm{H}$ protons showed signals in the downfield region compared to the benzenoid protons, with the 4'-H and 4"-H signals the most downfield. In the compounds having identical coumarin substitutions at 2- and 6-positions of the pyridine (compounds 3a-c and 3j-1), the 3-H and 5-H appeared as a singlet between $\delta$ 8.62-8.79. Similarly in these compounds, the 4'-H and 4"-H also gave a singlet in the region $\delta 8.86-9.05$. In the case of compounds $3 \mathbf{d}-\mathbf{i}$, the $3-\mathrm{H}$ and $5-\mathrm{H}$ gave two singlets in the region $\delta 8.55-8.64$, while $4 '-\mathrm{H}$ and 4"-H gave two singlets between $\delta$ 9.06-9.13. In the case of compounds 3m-r, the 3-H appeared as singlet at around $\delta 8.70$, while the 5-H appeared as singlet at around $\delta 8.60$. Similarly in these compounds the 4'-H appeared as singlet between $\delta$ 9.65-9.83, while 4"-H appeared as a singlet between $\delta 8.90-9.10$. It was possible to differentiate the $3-\mathrm{H}, 5-\mathrm{H}$ and $4^{\prime}-\mathrm{H}$, $4 "-H$ signals in these compounds 3m-r because of the presence of an additional fused benzene ring in the coumarin moiety at $\mathrm{C}-2$, which causes diamagnetic anisotropy and deshields the $3-\mathrm{H}$ and 4'-H compared to the 5-H and 4"-H.

The ${ }^{13} \mathrm{C}$ NMR spectra of compounds 3a-r showed expected signals for non equivalent carbons present in the compounds. Similarly the DEPT 90 spectra gave the expected non equivalent tertiary carbon signals.

The selected mass spectrum of compound 3a showed $\mathrm{M}^{+}$peak at $\mathrm{m} / \mathrm{z} 443$ (100\%) along with other fragment peaks. The appearance of a molecular ion peak at 443 mass units supports the structure of compound 3a.

\section{Antimicrobial activity}

All the synthesized compounds 3a-r were screened for their antibacterial and antifungal activity. Compounds 3a-r were screened against Escherichia coli (Gram-negative bacteria) and Bacillus subtilis (Gram-positive bacteria) and antifungal activity against Aspergillus niger (Fungi). The evaluation of antimicrobial activity was carried out using the agar cup diffusion method. The results are summarized in Table 1 . None of the compounds showed antifungal activity against fungi $A$. niger. All the compounds 3a-r showed moderate activity against the Gram-positive bacteria $B$. subtilis. The results towards this bacteria reveal that the incorporation of the substituent groups like $\mathrm{CH}_{3}$ or $\mathrm{OCH}_{3}$ either in the coumarin nucleus or in a phenyl ring does not affect the antibacterial activity much more and all the compounds have almost same activity. Compounds 3a-l show moderate activity towards Gram-negative bacteria E. coli, but compounds 3m-r are inactive. Thus the presence of an additional fused benzene ring between the C-5' and C6' positions inhibits the antibacterial activity towards E. coli.

Table 1. Antimicrobial activity of 4-aryl-2,6-di(coumarin-3-yl)pyridines 3a-r Compd. Inhibition zone in $\mathrm{mm}$ 


\begin{tabular}{cccc}
\hline & \multicolumn{2}{c}{ Bacteria } & Fungi \\
\cline { 2 - 4 } 3a & E. coli & B. subtilis & A. niger \\
3b & 14 & 11 & - \\
3c & 15 & 12 & - \\
3d & 12 & 12 & - \\
3e & 14 & 10 & - \\
3f & 14 & 12 & - \\
3g & 15 & 11 & - \\
3h & 14 & 10 & - \\
3i & 14 & 12 & - \\
3j & 15 & 11 & - \\
3k & 15 & 12 & - \\
3l & 13 & 12 & - \\
3m & - & 13 & - \\
3n & - & 12 & - \\
3o & - & 11 & - \\
3p & - & 12 & - \\
3q & - & 10 & - \\
3r & - & 12 & - \\
Ciprofloxacin & 18 & 25 & NT \\
Ampicillin & 20 & 27 & NT \\
Griseofulvin & NT & NT & 25 \\
\hline
\end{tabular}

- denotes: inhibition zone was not observed.

NT denotes: not tested.

\section{Conclusions}

We have developed a simple and convenient method for the synthesis of 4-aryl-2,6-dicoumarinyl pyridine derivatives utilizing the Kröhnke reaction. The method allows incorporation of identical or non-identical coumarin moieties at 2- and 6-positions of pyridine.

\section{Experimental Section}


General. The melting points reported are uncorrected. IR spectra ( $\mathrm{KBr}$ disc) were recorded on Shimadzu FT-IR 8400-S spectrometer. ${ }^{1} \mathrm{H}$ NMR and ${ }^{13} \mathrm{C}$ NMR spectra were recorded on a Bruker Avance 400 spectrometer operating at $400 \mathrm{MHz}$ for ${ }^{1} \mathrm{H} \mathrm{NMR}$ and $100 \mathrm{MHz}$ for ${ }^{13} \mathrm{C}$ NMR. The chemical shift $(\delta)$ is reported in ppm using $\mathrm{CDCl}_{3}$ as a solvent and a calibrated standard solvent signal. The mass spectrum of compound 3a was recorded on a Shimadzu QP 2010 spectrometer. 3-Coumarinoyl methyl pyridinium salts 1a-c and 1-[2H-1-benzopyran-2-on3-yl]-3-aryl-prop-2-en-1-ones 2a-f were prepared according literature procedures. ${ }^{14,15}$

\section{General procedure for the synthesis of 4-aryl-2,6-di(coumarin-3-yl)pyridines (3a-r)}

In a $100 \mathrm{~mL}$ three necked round bottom flask equipped with a dropping funnel, condenser, guard tube and magnetic needle, an appropriate 3-coumarinoyl methyl pyridinium salt 1a-c $(0.003 \mathrm{~mol})$ in glacial acetic acid $(15 \mathrm{~mL})$ was taken. To this, $\mathrm{NH}_{4} \mathrm{OAc}(0.03 \mathrm{~mol})$ was added with stirring at room temperature. Then a solution of an appropriate 1-[2H-1-benzopyran-2-on-3-yl]-3-arylprop-2-en-1-one 2a-f $(0.003 \mathrm{~mol})$ in glacial acetic acid $(15 \mathrm{~mL})$ was added with stirring at $\mathrm{rt}$ during $15 \mathrm{~min}$. The reaction mixture was further stirred for $1 \mathrm{~h}$ and then heated for $8 \mathrm{~h}$ at $140{ }^{\circ} \mathrm{C}$. It was then allowed to come to $\mathrm{rt}$ and poured into ice-cold water $(75 \mathrm{~mL})$. A crude solid obtained was extracted with chloroform $(3 \times 30 \mathrm{~mL})$. The organic layer was washed with $5 \% \mathrm{NaHCO}_{3}$ solution $(3 \times 20 \mathrm{~mL})$, water $(2 \times 20 \mathrm{~mL})$ and dried over anhydrous $\mathrm{Na}_{2} \mathrm{SO}_{4}$. The removal of chloroform under reduced pressure gave crude material which was subjected to column chromatography using silica gel and $\mathrm{CHCl}_{3}$-petroleum ether (60-80) (9:1) as an eluent to give appropriate product 3a-r. The compounds were recrystallized from $\mathrm{CHCl}_{3}$-hexane.

4-Phenyl-2,6-di(coumarin-3-yl)pyridine (3a). Yield 57\%; mp 253-254 ${ }^{\circ} \mathrm{C}$; white solid; IR (KBr): 3059 (m), 1713 (vs), 1607 (s), 1543 (s), 749 (s), 688 (s) cm ${ }^{-1} ;{ }^{1} \mathrm{H} \mathrm{NMR}\left(\mathrm{CDCl}_{3}\right): \delta$ 7.36$7.84(13 \mathrm{H}, \mathrm{m}, \mathrm{Ar}-\mathrm{H}), 8.66(2 \mathrm{H}, \mathrm{s}, 3-\mathrm{H}$ and $5-\mathrm{H}$ of pyridine ring), 8.89 (2H, s, 4'-H and 4"-H of coumarin rings); ${ }^{13} \mathrm{C}$ NMR: $\delta 116.4(\mathrm{CH}), 119.5(\mathrm{C}), 121.8(\mathrm{CH}), 124.6(\mathrm{CH}), 125.3(\mathrm{C}), 127.4$ $(\mathrm{CH}), 128.9(\mathrm{CH}), 129.1(\mathrm{CH}), 129.2(\mathrm{CH}), 132.3(\mathrm{CH}), 138.0(\mathrm{C}), 142.8(\mathrm{CH}), 149.8(\mathrm{C}), 151.4$ (C), 154.0 (C), 160.3 (C). Anal. Calcd. for $\mathrm{C}_{29} \mathrm{H}_{17} \mathrm{NO}_{4}$ : C, 78.55; H, 3.86; N, 3.16\%. Found: C, $78.46 ; \mathrm{H}, 3.94 ; \mathrm{N}, 3.25 \%$.

4-(4-Methylphenyl)-2,6-di(coumarin-3-yl)pyridine (3b). Yield 64\%; mp 269-270 ${ }^{\circ} \mathrm{C}$; light yellow solid; IR (KBr): 3055 (m), 1720 (vs), 1605 (s), 1530 (s), 825 (s) cm ${ }^{-1} ;{ }^{1} \mathrm{H}$ NMR: $\delta 2.43$ $\left(3 \mathrm{H}, \mathrm{s}, \mathrm{CH}_{3}\right), 7.30-7.76(12 \mathrm{H}, \mathrm{m}, \mathrm{Ar}-\mathrm{H}), 8.62(2 \mathrm{H}, \mathrm{s}, 3-\mathrm{H}$ and $5-\mathrm{H}$ of pyridine ring), $8.86(2 \mathrm{H}, \mathrm{s}$, 4'-H and 4"-H of coumarin rings); ${ }^{13} \mathrm{C}$ NMR: $\delta 21.3\left(\mathrm{CH}_{3}\right), 116.4(\mathrm{CH}), 119.5(\mathrm{CH}), 121.5(\mathrm{C})$, $124.6(\mathrm{CH}), 125.4(\mathrm{C}), 127.2(\mathrm{CH}), 128.9(\mathrm{CH}), 129.8(\mathrm{CH}), 132.3(\mathrm{CH}), 135.1(\mathrm{C}), 139.4(\mathrm{C})$, $142.8(\mathrm{CH}), 149.8(\mathrm{C}), 151.4(\mathrm{C}), 154.0(\mathrm{C}), 160.3(\mathrm{C})$. Anal. Calcd. for $\mathrm{C}_{30} \mathrm{H}_{19} \mathrm{NO}_{4}$ : C, 78.76; H, 4.19; N, 3.06\%. Found: C, 78.85; H, 4.12; N, 3.01\%.

4-(4-Methoxyphenyl)-2,6-di(coumarin-3-ylpyridine (3c). Yield 59\%; mp 277-278 ${ }^{\circ} \mathrm{C}$; light yellow solid; IR (KBr): 3060 (m), 1715 (vs), 1610 (s), 1510 (s), 825 (s) cm ${ }^{-1} ;{ }^{1} \mathrm{H}$ NMR: $\delta 3.90$ $\left(3 \mathrm{H}, \mathrm{s}, \mathrm{OCH}_{3}\right), 7.02-7.80(12 \mathrm{H}, \mathrm{m}, \mathrm{Ar}-\mathrm{H}), 8.62(2 \mathrm{H}, \mathrm{s}, 3-\mathrm{H}$ and $5-\mathrm{H}$ of pyridine ring), $8.88(2 \mathrm{H}$, s, 4'-H and 4"-H of coumarin rings); ${ }^{13} \mathrm{C}$ NMR: $\delta 55.5\left(\mathrm{OCH}_{3}\right), 113.9(\mathrm{C}), 114.5(\mathrm{CH}), 116.4$ $(\mathrm{CH}), 119.5(\mathrm{C}), 121.1(\mathrm{CH}), 124.6(\mathrm{CH}), 125.4(\mathrm{C}), 128.6(\mathrm{CH}), 128.9(\mathrm{CH}), 130.2(\mathrm{C}), 132.2$ 
(CH), $142.8(\mathrm{CH}), 151.3(\mathrm{C}), 154.0(\mathrm{C}), 160.3(\mathrm{C}), 160.7$ (C). Anal. Calcd. for $\mathrm{C}_{30} \mathrm{H}_{19} \mathrm{NO}_{5}$ : C, 76.10; H, 4.04; N, 2.96\%. Found: C, 76.01 H, 4.13; N, 2.96\%.

4-Phenyl-2-(coumarin-3-yl)-6-(8-methoxycoumarin-3-yl)pyridine (3d). Yield 55\%; mp >300 ${ }^{\circ} \mathrm{C}$; white solid; IR (KBr): 3055 (m), 1720 (vs), 1595 (s), 1495 (s), 755 (s), 695 (s) cm ${ }^{-1} ;{ }^{1} \mathrm{H}$ NMR: $\delta 4.06\left(3 \mathrm{H}, \mathrm{s}, \mathrm{OCH}_{3}\right), 7.41-7.91(12 \mathrm{H}, \mathrm{m}, \mathrm{Ar}-\mathrm{H}), 8.62$ and $8.63(2 \mathrm{H}$, two s, 3-H and 5-H of pyridine ring), 9.07 and $9.11\left(2 \mathrm{H}\right.$, two s, 4 '-H and 4 "-H of coumarin rings); ${ }^{13} \mathrm{C}$ NMR: $\delta 56.6$ $\left(\mathrm{OCH}_{3}\right), 114.2(\mathrm{C}), 114.4(\mathrm{C}), 117.1(\mathrm{CH}), 118.2(\mathrm{CH}), 118.6(\mathrm{C}), 118.9(\mathrm{C}), 121.1(\mathrm{CH}), 121.5$ $(\mathrm{CH}), 126.8(\mathrm{CH}), 127.8(\mathrm{CH}), 130.1(\mathrm{CH}), 130.6(\mathrm{CH}), 132.9(\mathrm{CH}), 136.7(\mathrm{CH}), 145.7(\mathrm{C})$, $145.8(\mathrm{C}), 147.2(\mathrm{C}), 148.3(\mathrm{CH}), 148.6(\mathrm{CH}), 154.1(\mathrm{C}), 158.9(\mathrm{C}), 161.3(\mathrm{C})$. Anal. Calcd. for $\mathrm{C}_{30} \mathrm{H}_{19} \mathrm{NO}_{5}$ : C, 76.10; H, 4.04; N, 2.96\%. Found: C, 76.01; H, 4.16; N, 2.84\%.

4-(4-Methylphenyl)-2-(coumarin-3-yl)-6-(8-methoxycoumarin-3-yl)pyridine (3e). Yield 61\%; mp 283-284 ${ }^{\circ} \mathrm{C}$; light yellow solid; IR (KBr): 3060 (m), 1715 (vs), 1595 (s), 1525 (s), 825 (s) $\mathrm{cm}^{-1}$; ${ }^{1} \mathrm{H}$ NMR: $\delta 2.45\left(3 \mathrm{H}, \mathrm{s}, \mathrm{CH}_{3}\right), 4.04\left(3 \mathrm{H}, \mathrm{s}, \mathrm{OCH}_{3}\right), 7.35-7.88(11 \mathrm{H}, \mathrm{m}, \mathrm{Ar}-\mathrm{H}), 8.61$ and $8.64(2 \mathrm{H}$, two s, 3-H and 5-H of pyridine ring), 9.07 and 9.13 (2H, two s, 4'-H and 4"-H of coumarin rings); ${ }^{13} \mathrm{C}$ NMR: $\delta 21.2\left(\mathrm{CH}_{3}\right), 56.6\left(\mathrm{OCH}_{3}\right), 116.0(\mathrm{CH}), 118.0(\mathrm{CH}), 118.9(\mathrm{C})$, $120.4(\mathrm{CH}), 120.5(\mathrm{CH}), 126.7(\mathrm{CH}), 127.9(\mathrm{CH}), 129.0(\mathrm{C}), 129.6(\mathrm{CH}), 130.2(\mathrm{CH}), 131.0(\mathrm{C})$, $139.1(\mathrm{CH}), 143.2(\mathrm{CH}), 143.6(\mathrm{C}), 144.5(\mathrm{C}), 145.4(\mathrm{C}), 146.0(\mathrm{C}), 147.3(\mathrm{C}), 148.5(\mathrm{CH})$, 155.4 (C), 158.5 (C), 161.2 (C). Anal. Calcd. for $\mathrm{C}_{31} \mathrm{H}_{21} \mathrm{NO}_{5}: \mathrm{C}, 76.38 ; \mathrm{H}, 4.34 ; \mathrm{N}, 2.87 \%$. Found: C, 76.48; H, 4.23; N, 2.79\%.

4-(4-Methoxyphenyl)-2-(coumarin-3-yl)-6-(8-methoxycoumarin-3-yl)pyridine (3f). Yield 56\%; mp 269-270 ${ }^{\circ} \mathrm{C}$; yellow solid; IR (KBr): 3055 (m), 1715 (vs), 1610 (s), 1515 (s), 830 (s) $\mathrm{cm}^{-1}$; ${ }^{1} \mathrm{H}$ NMR: $\delta 3.92\left(3 \mathrm{H}, \mathrm{s}, \mathrm{OCH}_{3}\right), 4.05\left(3 \mathrm{H}, \mathrm{s}, \mathrm{OCH}_{3}\right), 7.11-7.94(11 \mathrm{H}, \mathrm{m}, \mathrm{Ar}-\mathrm{H}), 8.55$ and $8.56(2 \mathrm{H}$, two s, 3-H and 5-H of pyridine ring), 9.06 and 9.09 (2H, two s, 4'-H and 4"-H of coumarin rings); ${ }^{13} \mathrm{C}$ NMR: $\delta 55.7\left(\mathrm{OCH}_{3}\right), 56.6\left(\mathrm{OCH}_{3}\right), 114.5(\mathrm{C}), 114.7(\mathrm{C}), 115.7(\mathrm{CH})$, $117.0(\mathrm{CH}), 118.0(\mathrm{CH}), 118.2(\mathrm{C}), 118.9(\mathrm{C}), 119.7(\mathrm{CH}), 121.5(\mathrm{CH}), 125.7(\mathrm{C}), 126.7(\mathrm{CH})$, $129.8(\mathrm{CH}), 130.5(\mathrm{CH}), 136.5(\mathrm{CH}), 143.6(\mathrm{C}), 145.3(\mathrm{C}), 145.4(\mathrm{C}), 147.2(\mathrm{C}), 148.0(\mathrm{CH})$, 148.2 (CH), 154.1 (C), 157.7 (C), 163.9 (C). Anal. Calcd. for $\mathrm{C}_{31} \mathrm{H}_{21} \mathrm{NO}_{6}$ : C, 73.95; H, 4.20; $\mathrm{N}$, 2.78\%. Found: C, 73.87; H, 4.13; N, 2.78\%.

4-Phenyl-2-(8-methoxycoumarin-3-yl)-6-(coumarin-3-yl)pyridine (3g). Yield 64\%; mp >300 ${ }^{\circ} \mathrm{C}$; white solid; IR (KBr): 3055 (m), 1720 (vs), 1595 (s), 1550 (s), 755 (s), 690 (s) cm ${ }^{-1} ;{ }^{1} \mathrm{H}$ NMR: $\delta 4.06\left(3 \mathrm{H}, \mathrm{s}, \mathrm{OCH}_{3}\right), 7.41-7.91(12 \mathrm{H}, \mathrm{m}, \mathrm{Ar}-\mathrm{H}), 8.62$ and $8.63(2 \mathrm{H}$, two s, 3-H and 5-H of pyridine ring), 9.07 and $9.11\left(2 \mathrm{H}\right.$, two s, 4'-H and 4"-H of coumarin rings); ${ }^{13} \mathrm{C}$ NMR: $\delta 56.6$ $\left(\mathrm{OCH}_{3}\right), 114.2(\mathrm{C}), 114.4(\mathrm{C}), 117.1(\mathrm{CH}), 118.2(\mathrm{CH}), 118.6(\mathrm{C}), 118.9(\mathrm{C}), 121.1(\mathrm{CH}), 121.5$ $(\mathrm{CH}), 126.8(\mathrm{CH}), 127.8(\mathrm{CH}), 130.1(\mathrm{CH}), 130.6(\mathrm{CH}), 132.9(\mathrm{CH}), 136.7(\mathrm{CH}), 145.7(\mathrm{C})$, $145.8(\mathrm{C}), 147.2(\mathrm{C}), 148.3(\mathrm{CH}), 148.6(\mathrm{CH}), 154.1(\mathrm{C}), 158.7(\mathrm{C}), 161.3(\mathrm{C})$. Anal. Calcd. for $\mathrm{C}_{30} \mathrm{H}_{19} \mathrm{NO}_{5}$ : C, 76.10; H, 4.04; N, 2.96\%. Found: C, 76.01; H, 4.16; N, 2.84\%.

4-(4-Methylphenyl)-2-(8-methoxycoumarin-3-yl)-6-(coumarin-3-yl)pyridine (3h). Yield 59\%; mp 283-284 ${ }^{\circ} \mathrm{C}$; light yellow solid; IR (KBr): 3060 (m), 1715 (vs), 1595 (s), 1525 (s), 825 (s) $\mathrm{cm}^{-1}$; ${ }^{1} \mathrm{H}$ NMR: $\delta 2.45\left(3 \mathrm{H}, \mathrm{s}, \mathrm{CH}_{3}\right), 4.04\left(3 \mathrm{H}, \mathrm{s}, \mathrm{OCH}_{3}\right), 7.35-7.88(11 \mathrm{H}, \mathrm{m}, \mathrm{Ar}-\mathrm{H}), 8.61$ and $8.64(2 \mathrm{H}$, two s, 3-H and 5-H of pyridine ring), 9.07 and $9.13(2 \mathrm{H}$, two s, 4'-H and 4"-H of 
coumarin rings); ${ }^{13} \mathrm{C}$ NMR: $\delta 21.2\left(\mathrm{CH}_{3}\right), 56.6\left(\mathrm{OCH}_{3}\right), 116.0(\mathrm{CH}), 118.0(\mathrm{CH}), 118.9(\mathrm{C})$, $120.4(\mathrm{CH}), 120.5(\mathrm{CH}), 126.7(\mathrm{CH}), 127.9(\mathrm{CH}), 129.0(\mathrm{C}), 129.6(\mathrm{CH}), 130.2(\mathrm{CH}), 131.0(\mathrm{C})$, $139.1(\mathrm{CH}), 143.2(\mathrm{CH}), 143.6(\mathrm{C}), 144.5(\mathrm{C}), 145.4(\mathrm{C}), 146.0(\mathrm{C}), 147.3(\mathrm{C}), 148.5(\mathrm{CH})$, 155.4 (C), 158.5 (C), 161.2 (C). Anal. Calcd. for $\mathrm{C}_{31} \mathrm{H}_{21} \mathrm{NO}_{5}$ : C, 76.38; H, 4.34; N, 2.87\%. Found: C, 76.29; H, 4.26; N, 2.81\%.

(4-Methoxyphenyl)-2-(8-methoxycoumarin-3-yl)-6-(coumarin-3-yl)pyridine (3i). Yield 57\%; mp 269-270 ${ }^{\circ} \mathrm{C}$; yellow solid; IR (KBr): 3055 (m), 1715 (vs), 1610 (s), 1515 (s), 830 (s) cm ${ }^{-1} ;{ }^{1} \mathrm{H}$ NMR: $\delta 3.92\left(3 \mathrm{H}, \mathrm{s}, \mathrm{OCH}_{3}\right), 4.05\left(3 \mathrm{H}, \mathrm{s}, \mathrm{OCH}_{3}\right), 7.11-7.94(11 \mathrm{H}, \mathrm{m}, \mathrm{Ar}-\mathrm{H}), 8.55$ and $8.56(2 \mathrm{H}$, two s, 3-H and 5-H of pyridine ring), 9.06 and 9.09 (2H, two s, 4'-H and 4"-H of coumarin rings); ${ }^{13} \mathrm{C}$ NMR: $\delta 55.7\left(\mathrm{OCH}_{3}\right), 56.6\left(\mathrm{OCH}_{3}\right), 114.5(\mathrm{C}), 114.7(\mathrm{C}), 115.9(\mathrm{CH}), 117.0(\mathrm{CH})$, $118.0(\mathrm{CH}), 118.2(\mathrm{C}), 118.9(\mathrm{C}), 119.7(\mathrm{CH}), 121.5(\mathrm{CH}), 125.7(\mathrm{C}), 126.7(\mathrm{CH}), 129.8(\mathrm{CH})$, $130.5(\mathrm{CH}), 136.5(\mathrm{CH}), 143.6(\mathrm{C}), 145.3(\mathrm{C}), 145.4(\mathrm{C}), 147.2(\mathrm{C}), 148.0(\mathrm{CH}), 148.2(\mathrm{CH})$, 154.1 (C), 157.7 (C), 163.9 (C). Anal. Calcd. for $\mathrm{C}_{31} \mathrm{H}_{21} \mathrm{NO}_{6}: \mathrm{C}, 73.95 ; \mathrm{H}, 4.20 ; \mathrm{N}, 2.78 \%$. Found: C, $73.87 ; \mathrm{H}, 4.13 ; \mathrm{N}, 2.87 \%$.

4-Phenyl-2,6-di(8-methoxycoumarin-3-yl)pyridine (3j). Yield 56\%; mp 280-281 ${ }^{\circ} \mathrm{C}$; light yellow solid; IR(KBr): 3055 (m), 1720 (vs), 1610 (s), 1550 (s), 755 (s), 700 (s) cm ${ }^{-1}$; ${ }^{1} \mathrm{H}$ NMR: $\delta$ $4.06\left(6 \mathrm{H}, \mathrm{s}, 2 \mathrm{x} \mathrm{OCH}_{3}\right), 7.51-7.94(11 \mathrm{H}, \mathrm{m}, \mathrm{Ar}-\mathrm{H}), 8.79(2 \mathrm{H}, \mathrm{s}, 3-\mathrm{H}$ and 5-H of pyridine ring), $9.05\left(2 \mathrm{H}, \mathrm{s}, 4{ }^{\prime}-\mathrm{H}\right.$ and 4 "-H of coumarin rings); ${ }^{13} \mathrm{C} \mathrm{NMR:} \delta 56.5\left(\mathrm{OCH}_{3}\right), 116.5(\mathrm{CH}), 119.4(\mathrm{C})$, $121.9(\mathrm{CH}), 124.6(\mathrm{CH}), 125.5(\mathrm{C}), 127.4(\mathrm{CH}), 129.0(\mathrm{CH}), 129.1(\mathrm{CH}), 132.4(\mathrm{CH}), 138.0(\mathrm{C})$, $142.8(\mathrm{CH}), 147.1(\mathrm{C}), 149.8(\mathrm{C}), 151.3(\mathrm{C}), 154.0(\mathrm{C}), 160.3(\mathrm{C})$. Anal. Calcd. for $\mathrm{C}_{31} \mathrm{H}_{21} \mathrm{NO}_{6}$ : C, 73.95; H, 4.20; N, 2.78\%. Found: C, 73.85; H, 4.32; N, 2.68\%.

4-(4-Methylphenyl)-2,6-di(8-methoxycoumarin-3-yl)pyridine (3k). Yield 58\%; mp 282-283 ${ }^{\circ} \mathrm{C}$; yellow solid; IR (KBr): 3060 (m), 1715 (vs), 1610 (s), 1540 (s), 825 (s) cm ${ }^{-1}$; ${ }^{1} \mathrm{H}$ NMR: $\delta$ $2.46\left(3 \mathrm{H}, \mathrm{s}, \mathrm{CH}_{3}\right), 3.91\left(6 \mathrm{H}, \mathrm{s}, 2 \times \mathrm{OCH}_{3}\right), 7.41-7.92(10 \mathrm{H}, \mathrm{m}, \mathrm{Ar}-\mathrm{H}), 8.77(2 \mathrm{H}, \mathrm{s}, 3-\mathrm{H}$ and $5-\mathrm{H}$ of pyridine ring), $9.04\left(2 \mathrm{H}, \mathrm{s}, 4\right.$ '-H and 4 "- $\mathrm{H}$ of coumarin rings); ${ }^{13} \mathrm{C} \mathrm{NMR}: 21.3\left(\mathrm{CH}_{3}\right), 56.6$ $\left(\mathrm{OCH}_{3}\right), 116.5(\mathrm{CH}), 121.6(\mathrm{C}), 124.5(\mathrm{CH}), 125.5(\mathrm{C}), 127.3(\mathrm{CH}), 129.0(\mathrm{CH}), 129.9(\mathrm{CH})$, $132.2(\mathrm{CH}), 135.0(\mathrm{C}), 139.4(\mathrm{C}), 142.8(\mathrm{CH}), 147.0(\mathrm{C}), 149.9(\mathrm{C}), 151.5(\mathrm{C}), 154.0(\mathrm{C}), 160.30$ (C). Anal. Calcd. for $\mathrm{C}_{32} \mathrm{H}_{23} \mathrm{NO}_{6}$ : C, 74.27; H, 4.48; N, 2.71\%. Found: C, 74.20; H, 4.54; N, $2.84 \%$.

4-(4-Methoxyphenyl)-2,6-di(8-methoxycoumarin-3-yl)pyridine (3l). Yield 59\%; mp 274-275 ${ }^{\circ} \mathrm{C}$; light yellow solid; IR (KBr): 3050 (m), 1715 (vs), 1605 (s), 1555 (s), 830 (s) cm ${ }^{-1}$; ${ }^{1} \mathrm{H}$ NMR: $\delta 3.91\left(3 \mathrm{H}, \mathrm{s}, \mathrm{OCH}_{3}\right), 4.04\left(6 \mathrm{H}, \mathrm{s}, 2 \mathrm{x} \mathrm{OCH}_{3}\right), 7.10-7.91(10 \mathrm{H}, \mathrm{m}, \mathrm{Ar}-\mathrm{H}), 8.78(2 \mathrm{H}, \mathrm{s}, 3-\mathrm{H}$ and 5$\mathrm{H}$ of pyridine ring), $9.04\left(2 \mathrm{H}, \mathrm{s}, 4 \mathrm{\prime}-\mathrm{H}\right.$ and $4 "-\mathrm{H}$ of coumarin rings); ${ }^{13} \mathrm{C} \mathrm{NMR}: \delta 55.5\left(\mathrm{OCH}_{3}\right)$, $56.6\left(\mathrm{OCH}_{3}\right), 114.0(\mathrm{C}), 114.6(\mathrm{CH}), 119.6(\mathrm{C}), 121.2(\mathrm{CH}), 124.7(\mathrm{CH}), 125.4(\mathrm{C}), 128.6(\mathrm{CH})$, $129.0(\mathrm{CH}), 130.1(\mathrm{C}), 132.3(\mathrm{CH}), 142.8(\mathrm{CH}), 147.3(\mathrm{C}), 151.2(\mathrm{C}), 153.9(\mathrm{C}), 160.3(\mathrm{C})$, 160.7 (C). Anal. Calcd. for $\mathrm{C}_{32} \mathrm{H}_{23} \mathrm{NO}_{7}$ : C, 72.04; H, 4.35; N, 2.63\%. Found: C, 72.18; H, 4.48; $\mathrm{N}, 2.75 \%$.

4-Phenyl-2-(benzo[f]coumarin-3-yl)-6-(coumarin-3-yl)pyridine (3m). Yield 65\%; mp 265$266{ }^{\circ} \mathrm{C}$; yellow solid; IR (KBr): $3055(\mathrm{~m}), 1725$ (vs), 1610 (s), 1560 (s), 755(s), 695 (s) $\mathrm{cm}^{-1} ;{ }^{1} \mathrm{H}$ NMR: $\delta$ 7.36-8.50 $(15 \mathrm{H}, \mathrm{m}, \mathrm{Ar}-\mathrm{H}), 8.64(1 \mathrm{H}, \mathrm{s}, 5-\mathrm{H}$ of pyridine ring $), 8.72(1 \mathrm{H}, \mathrm{s}, 3-\mathrm{H}$ of 
pyridine ring), $8.90\left(1 \mathrm{H}\right.$, s, 4"-H of coumarin ring), $9.65(1 \mathrm{H}$, s, 4 '- $\mathrm{H}$ of coumarin ring $) ;{ }^{13} \mathrm{C}$ NMR: $\delta 113.8(\mathrm{C}), 116.5(\mathrm{CH}), 116.6(\mathrm{CH}), 119.6(\mathrm{C}), 121.7(\mathrm{CH}), 121.8(\mathrm{CH}), 121.8(\mathrm{CH})$, $124.2(\mathrm{C}), 124.6(\mathrm{CH}), 125.6(\mathrm{C}), 126.2(\mathrm{CH}), 127.4(\mathrm{CH}), 128.7(\mathrm{CH}), 128.9(\mathrm{CH}), 129.0(\mathrm{CH})$, $129.1(\mathrm{CH}), 129.2(\mathrm{CH}), 129.6(\mathrm{C}), 130.4(\mathrm{C}), 132.3(\mathrm{CH}), 133.7(\mathrm{CH}), 138.1(\mathrm{C}), 138.4(\mathrm{CH})$, 142.7 (CH), 149.8 (C), $151.6(\mathrm{C}), 151.8$ (C), 154.0 (C), 154.1 (C), 160.3 (C), 160.4 (C). Anal. Calcd. for $\mathrm{C}_{33} \mathrm{H}_{19} \mathrm{NO}_{4}$ : C, 80.31; H, 3.88; N, 2.84\%. Found: C, 80.25; H, 3.75; N, 2.96\%.

4-(4-Methylphenyl)-2-(benzo[f]coumarin-3-yl)-6-(coumarin-3-yl)pyridine (3n). Yield 66\%; mp 287-288 ${ }^{\circ} \mathrm{C}$; yellow solid; IR(KBr): 3050 (m), 1730 (vs), 1610 (s), 1520 (s), 825 (s) cm ${ }^{-1} ;{ }^{1} \mathrm{H}$ NMR: $2.44\left(3 \mathrm{H}, \mathrm{s}, \mathrm{CH}_{3}\right), 7.31-8.51(14 \mathrm{H}, \mathrm{m}, \mathrm{Ar}-\mathrm{H}), 8.63(1 \mathrm{H}, \mathrm{s}, 5-\mathrm{H}$ of pyridine ring), $8.71(1 \mathrm{H}$, s, 3-H of pyridine ring), $8.90(1 \mathrm{H}, \mathrm{s}, 4$ "- $\mathrm{H}$ of coumarin ring), $9.65(1 \mathrm{H}, \mathrm{s}, 4$ '-H of coumarin ring); ${ }^{13} \mathrm{C}$ NMR: $\delta 21.3\left(\mathrm{CH}_{3}\right), 113.8(\mathrm{C}), 116.5(\mathrm{CH}), 116.7(\mathrm{CH}), 119.6(\mathrm{C}), 121.5(\mathrm{CH}), 121.6(\mathrm{CH})$, $121.9(\mathrm{CH}), 124.3(\mathrm{C}), 124.6(\mathrm{CH}), 125.7(\mathrm{C}), 126.2(\mathrm{CH}), 127.3(\mathrm{CH}), 128.5(\mathrm{CH}), 128.9(\mathrm{CH})$, $129.1(\mathrm{CH}), 129.6(\mathrm{C}), 129.8(\mathrm{CH}), 130.4(\mathrm{C}), 132.2(\mathrm{CH}), 133.7(\mathrm{CH}), 135.2(\mathrm{C}), 138.5(\mathrm{CH})$, 139.4 (C), 142.7 (CH), 149.8 (C), 151.5 (C), 151.7 (C), 154.0 (C), 154.0 (C), 160.3 (C), 160.4 (C). Anal. Calcd. for $\mathrm{C}_{34} \mathrm{H}_{21} \mathrm{NO}_{4}$ : C, 80.46; H, 4.17; N, 2.76\%. Found: C, 80.33; H, 4.07; N, $2.84 \%$.

4-(4-Methoxyphenyl)-2-(benzo[f]coumarin-3-yl)-6-(coumarin-3-yl)pyridine (3o). Yield 62\%; mp 281-282 ${ }^{\circ} \mathrm{C}$; yellow solid; IR (KBr): 3055 (m), 1720 (vs), 1605 (s), 1540 (s), 830 (s) cm ${ }^{-1} ;{ }^{1} \mathrm{H}$ NMR: $\delta 3.91\left(3 \mathrm{H}, \mathrm{s}, \mathrm{OCH}_{3}\right), 7.04-8.51(14 \mathrm{H}, \mathrm{m}, \mathrm{Ar}-\mathrm{H}), 8.62(1 \mathrm{H}, \mathrm{s}, 5-\mathrm{H}$ of pyridine ring $), 8.70$ $(1 \mathrm{H}, \mathrm{s}, 3-\mathrm{H}$ of pyridine ring), $8.91(1 \mathrm{H}, \mathrm{s}, 4$ "-H of coumarin ring), $9.66(1 \mathrm{H}, \mathrm{s}, 4$ '-H of coumarin ring); ${ }^{13} \mathrm{C}$ NMR: $\delta 55.7\left(\mathrm{OCH}_{3}\right), 110.0(\mathrm{C}), 112.8(\mathrm{C}), 115.6(\mathrm{C}), 115.7(\mathrm{CH}), 116.1(\mathrm{CH}), 117.2$ $(\mathrm{CH}), 118.3(\mathrm{C}), 118.5(\mathrm{C}), 119.5(\mathrm{C}), 119.6(\mathrm{C}), 119.8(\mathrm{CH}), 121.6(\mathrm{CH}), 126.9(\mathrm{CH}), 128.0$ $(\mathrm{CH}), 129.0(\mathrm{C}), 129.8(\mathrm{CH}), 129.9(\mathrm{CH}), 130.1(\mathrm{CH}), 130.6(\mathrm{CH}), 130.9(\mathrm{C}), 134.3(\mathrm{C}), 136.7$ $(\mathrm{CH}), 141.6(\mathrm{CH}), 143.4(\mathrm{CH}), 144.7(\mathrm{C}), 145.8(\mathrm{C}), 154.1(\mathrm{C}), 155.4(\mathrm{C}), 160.2(\mathrm{C}), 160.4(\mathrm{C})$. Anal. Calcd. for $\mathrm{C}_{34} \mathrm{H}_{21} \mathrm{NO}_{5}$ : C, 78.00; H, 4.04; N, 2.68\%. Found: C, 78.11; H, 4.17; N, 2.59\%.

4-Phenyl-2-(benzo[f]coumarin-3-yl)-6-(8-methoxycoumarin-3-yl)pyridine (3p). Yield 63\%; mp >300 ${ }^{\circ} \mathrm{C}$; yellow solid; IR(KBr): 3050 (m), 1725 (vs), 1610 (s), 1545 (s), 750 (s), 700 (s), cm ${ }^{1}$; ${ }^{1} \mathrm{H}$ NMR: $\delta 4.08\left(3 \mathrm{H}, \mathrm{s}, \mathrm{OCH}_{3}\right), 7.39-8.58(14 \mathrm{H}, \mathrm{m}, \mathrm{Ar}-\mathrm{H}), 8.63(1 \mathrm{H}, \mathrm{s}, 5-\mathrm{H}$ of pyridine ring), $8.82(1 \mathrm{H}, \mathrm{s}, 3-\mathrm{H}$ of pyridine ring), $9.10(1 \mathrm{H}, \mathrm{s}, 4 "-\mathrm{H}$ of coumarin ring $), 9.83(1 \mathrm{H}, \mathrm{s}, 4$ '-H of coumarin ring); ${ }^{13} \mathrm{C}$ NMR: $\delta 56.5\left(\mathrm{OCH}_{3}\right), 112.2(\mathrm{C}), 113.7(\mathrm{C}), 114.4(\mathrm{C}), 116.0(\mathrm{CH}), 118.4$ $(\mathrm{CH}), 118.9(\mathrm{C}), 120.9(\mathrm{CH}), 120.9(\mathrm{CH}), 121.3(\mathrm{CH}), 121.5(\mathrm{CH}), 127.0(\mathrm{CH}), 127.8(\mathrm{CH})$, $128.0(\mathrm{CH}), 128.9(\mathrm{C}), 129.8(\mathrm{CH}), 130.1(\mathrm{CH}), 130.4(\mathrm{CH}), 130.9(\mathrm{C}), 133.0(\mathrm{CH}), 134.0(\mathrm{C})$, $139.6(\mathrm{CH}), 143.5(\mathrm{CH}), 145.6(\mathrm{C}), 146.1(\mathrm{C}), 147.1(\mathrm{C}), 148.7(\mathrm{CH}), 155.5(\mathrm{C}), 159.1(\mathrm{C})$, 161.8 (C). Anal. Calcd. for $\mathrm{C}_{34} \mathrm{H}_{21} \mathrm{NO}_{5}$ : C, 78.00; H, 4.04; N, 2.68\%. Found: C, 78.12; H, 4.15; $\mathrm{N}, 2.58 \%$.

4-(4-Methylphenyl)-2-(benzo[f]coumarin-3-yl)-6-(8-methoxycoumarin-3-yl)pyridine (3q). Yield 55\%; mp >300 ${ }^{\circ}$; yellow solid; IR (KBr): 3060 (m), 1715 (vs), 1600 (s), 1550 (s), 820 (s) $\mathrm{cm}^{-1}$; ${ }^{1} \mathrm{H}$ NMR: $\delta 2.46\left(3 \mathrm{H}, \mathrm{s}, \mathrm{CH}_{3}\right), 4.05\left(3 \mathrm{H}, \mathrm{s}, \mathrm{OCH}_{3}\right), 7.37-8.59(14 \mathrm{H}, \mathrm{m}, \mathrm{Ar}-\mathrm{H}+5-\mathrm{H}$ of pyridine ring), $8.78(1 \mathrm{H}, \mathrm{s}, 3-\mathrm{H}$ of pyridine ring), $9.06(1 \mathrm{H}, \mathrm{s}, 4 "-\mathrm{H}$ of coumarin ring $), 9.78(1 \mathrm{H}$, s, 4'-H of coumarin ring); ${ }^{13} \mathrm{C}$ NMR: $\delta 21.3\left(\mathrm{CH}_{3}\right), 56.6\left(\mathrm{OCH}_{3}\right), 112.7(\mathrm{C}), 113.6(\mathrm{C}), 114.6$ 
(C), $116.0(\mathrm{CH}), 118.0(\mathrm{CH}), 118.9(\mathrm{C}), 120.4(\mathrm{CH}), 120.6(\mathrm{CH}), 121.6(\mathrm{CH}), 126.7(\mathrm{CH}), 127.8$ $(\mathrm{CH}), 129.0(\mathrm{C}), 129.7(\mathrm{CH}), 130.3(\mathrm{CH}), 130.8(\mathrm{CH}), 131.0(\mathrm{C}), 139.0(\mathrm{CH}), 143.2(\mathrm{CH}), 143.6$ (C), 144.4 (C), 145.5 (C), 145.9 (C), 147.8 (C), $148.4(\mathrm{CH}), 155.4$ (C), 158.5 (C), 161.2 (C). Anal. Calcd. for $\mathrm{C}_{35} \mathrm{H}_{23} \mathrm{NO}_{5}$ : C, 78.20; H, 4.31; N, 2.61\%. Found: C, 78.14; H, 4.22; N, 2.54\%.

4-(4-Methoxyphenyl)-2-(benzo[f]coumarin-3-yl)-6-(8-methoxycoumarin-3-yl)pyridine (3r). Yield 66\%; mp >300 ${ }^{\circ} \mathrm{C}$; yellow solid; IR(KBr): 3060 (m), 1715 (vs), 1610 (s), 1550 (s), 825 (s) $\mathrm{cm}^{-1}$; ${ }^{1} \mathrm{H}$ NMR: $\delta 3.95\left(3 \mathrm{H}, \mathrm{s}, \mathrm{OCH}_{3}\right), 4.07\left(3 \mathrm{H}, \mathrm{s}, \mathrm{OCH}_{3}\right), 7.15-8.59\left(14 \mathrm{H}, \mathrm{m}, \mathrm{Ar}-\mathrm{H}+\mathrm{C}_{5}-\mathrm{H}\right.$ of pyridine ring), $8.73(1 \mathrm{H}, \mathrm{s}, 3-\mathrm{H}$ of pyridine ring), $9.08(1 \mathrm{H}, \mathrm{s}, 4$ "- $\mathrm{H}$ of coumarin ring), $9.79(1 \mathrm{H}$, s, 4'-H of coumarin ring); ${ }^{13} \mathrm{C}$ NMR: $\delta 55.7\left(\mathrm{OCH}_{3}\right), 56.6\left(\mathrm{OCH}_{3}\right), 113.7(\mathrm{C}), 114.7(\mathrm{C}), 116.1$ $(\mathrm{CH}), 118.0(\mathrm{CH}), 119.0(\mathrm{C}), 119.8(\mathrm{CH}), 119.9(\mathrm{CH}), 121.6(\mathrm{CH}), 121.7(\mathrm{CH}), 125.9(\mathrm{C}), 126.8$ $(\mathrm{CH}), 127.9(\mathrm{CH}), 129.1(\mathrm{C}), 129.7(\mathrm{CH}), 129.9(\mathrm{CH}), 130.3(\mathrm{CH}), 130.8(\mathrm{C}), 139.0(\mathrm{CH}), 143.3$ $(\mathrm{CH}), 143.6(\mathrm{C}), 145.3(\mathrm{C}), 145.7(\mathrm{C}), 147.2(\mathrm{C}), 148.4(\mathrm{CH}), 155.4(\mathrm{C}), 161.5(\mathrm{C}), 163.8(\mathrm{C})$. Anal. Calcd. for $\mathrm{C}_{35} \mathrm{H}_{23} \mathrm{NO}_{6}$ : C, 75.94; H, 4.19; N, 2.53\%. Found: C, C, 75.85; H, 4.10; N, $2.68 \%$.

\section{Antimicrobial activity}

All the synthesized compounds 3a-r were screened for their antibacterial activities against Escherichia coli (Gram-negative bacteria), Bacillus subtilis (Gram-positive bacteria) and antifungal activity against Aspergillus niger (Fungi) by the agar cup diffusion method. ${ }^{16}$ The zone of inhibition was measured in mm and was compared with standard drug. DMF was used as blank, Ciprofloxacin and Ampicillin were used as antibacterial standards and Griseofulvin was used as antifungal standard. All the compounds were tested at $1000 \mu \mathrm{g} / \mathrm{ml}$ concentration.

\section{Acknowledgements}

The authors are thankful to the Head, Department of Chemistry, Sardar Patel University for providing research facilities and Vaibhav Laboratory, Ahmedabad for recording IR spectra.

\section{References}

1. Manolov, I.; Maichle-Moessmer, C.; Danchev, N. Eur. J. Med. Chem. 2006, 41, 882.

2. Khan, I.; Kulkarni, M.; Sun, C. Eur. J. Med. Chem. 2005, 40, 1168.

3. Nawrot-Morankaa, J.; Nawrotb, E.; Graczykb, J. Eur. J. Med. Chem. 2006, 41, 1301.

4. Reddy, N.; Reddy, M. M.; Cosenza, S.; Gumireddy, K.; Bell, C. S.; Reddy, P.; Reddy, M. R. Bioorg. Med. Chem. Lett. 2004, 14, 4093.

5. Donglei, Y.; Suzuki, M.; Lan, X.; Morris-Natschke, S.; Lee, K. Med. Res. Rev. 2003, 23, 322.

6. Moffett, R. B. U. S. Patent, 3156 697, 1964; Chem. Abstr. 1965, 62, $5257 f$. 
7. Sreenivasulu, B.; Sundaramurthy, V.; Subba, R. N. V. Proc. Ind. Acad. Sci., Sec. A, 1974, 79, 41.

8. Moffett, R. B., U. S. Patent, 3201 406, 1965; Chem. Abstr. 1965, 63, $13220 f$.

9. Moffett, R. B. J. Med. Chem. 1964, 7, 446.

10. (a) Brahmbhatt, D. I.; Raolji, G. B.; Pandya, S. U.; Pandya, U. R. Ind. J. Chem. 1999, 38B, 212. (b) Brahmbhatt, D. I.; Pandya, U. R. Ind. J. Chem. 2001, 40B, 419. (c) Brahmbhatt, D. I.; Pandya, U. R. Ind. J. Chem. 2003, 42B, 145. (d) Brahmbhatt, D. I.; Patel, C. N.; Pandya, V. P.; Patel, M. A. Ind. J. Chem. 2004, 43B, 2228. (e) Brahmbhatt, D. I.; Pandya, V. P.; Patel, C. N.; Patel, M. A. Ind. J. Chem. 2005, 44B, 1863. (f) Brahmbhatt, D. I.; Gajera, J. M.; Pandya, V. P.; Patel, M. A. Ind. J. Chem. 2007, 46B, 869. (g) Patel, N. H.; Patel, A. K.; Patel, C. V.; Patel, A. A.; Brahmbhatt, D. I. Arkivoc 2010, 2, 283.

11. El-Taweel, F. M. A.; Elnagdi, M. H. J. Heterocycl. Chem. 2001, 38, 981.

12. Verma, A. K.; Koul, S.; Kapoor, K. K.; Razdan, T. K. Aust. J. Chem. 2007, 60, 883.

13. (a) Krohnke, F.; Zecher, W. Chem. Ber. 1961, 94, 690. (b) Krohnke, F. Synthesis. 1976, 1, 1.

14. (a) Koelsch, C. F. J. Am. Chem. Soc 1950, 72, 2993. (b) Rao, T. V. P.; Rao, V. R. Ind. J. Chem. 1986, 25B, 413. (c) Skripskaya, O. V.; Yagodinets, P. I.; Chernyuk, I. N.; Shevchuk, M. I. Zh. Obshch. Khim. 1992, 62, 661.

15. Dimitrova, E.; Anghelova, Y. Synth. Commun. 1986, 16, 1195.

16. Copper, K. E.; Kavanagh, F. Analytical Microbiology; $2^{\text {nd }}$ Edn.; Academic Press: New York, 1972, pp 13. 